\title{
浅析倾斜摄影测量技术在不动产测绘中的应用
}

\author{
张萍 \\ 山东金桥建设项目管理有限公司，山东滩坊 262700
}

[摘要] 在社会快速发展的带动下, 为科学技术的发展带来了良好的机遇, 从而使得大量的新型科学技术被人们研发出来, 在 实践运用过程中取得了良好的成绩。就以往的不动产测绘工作来说, 涉及到的工作量较为巨大, 并且测绘工作整体效果较差, 极易受到外界多方面因素的影响。倾斜摄影测量技术是当前最为先进的一种测量专业技术, 其实质就是运用无人机技术来获 取需要的信息数据, 从而有效的避免危险事故的发生, 提升不动产测绘工作的整体效率和效果。

[关键词]倾斜摄影测量技术; 不动产测绘; 应用

DOI：10.33142/sca.v4i2.3802 中图分类号：P231 文献标识码：A

\section{Brief Analysis of Application of Oblique Photogrammetry in Real Estate Surveying and Mapping}

\section{ZHANG Ping}

Shandong Jinqiao Construction Project Management Co., Ltd., Weifang, Shandong, 262700, China

\begin{abstract}
Driven by the rapid development of society, it has brought good opportunities for the development of science and technology, so that a large number of new science and technology have been developed and good results have been achieved in the process of practical application. As for the previous real estate surveying and mapping work, the workload involved is huge and the overall effect of Surveying and mapping work is poor, which is easily affected by many external factors. Oblique photogrammetry technology is the most advanced measurement professional technology, its essence is to use UAV technology to obtain the required information data, so as to effectively avoid the occurrence of dangerous accidents and improve the overall efficiency and effect of real estate surveying and mapping work.
\end{abstract}

Keywords: oblique photogrammetry technology; real estate surveying and mapping; application

\section{引言}

近年来, 我国科学技术在多方面利好因素的影响下得到了显著的发展, 从而促进了倾斜摄影测量技术水平的不断 提升, 因为其具有较强的优越性所以被人们切实的运用到了不动产测绘工作之中。倾斜摄影测量技术其实质就是借助 多个传感器的共同作用, 在有限的时间内获取需要的信息数据, 将物体的实际情况进行全面的反应, 这种测绘方法与 之前的航拍技术测量模式存在明显的差别, 其在确保测量数据准确性方面具有良好的作用。

\section{1 倾斜摄影测量技术概念}

(1) 就倾斜摄影测量技术的实质来看, 其属于当前最为先进的一种科学技术方法, 在摄影装置中安设的传感器具 有一定的特殊性, 四个倾斜的角度与一个纵向角度相互配合, 这样就可以实现从多个角度进行拍摄的目的, 并且将这 项技术加以实践运用能够切实的对处理速度、高度以及飞行的方向加以记录。倾斜摄影测量技术的运用还需要借助飞 行器、POS 系统以及倾斜相机的辅助, 将飞行器加以实践运用可以完成对各类设备的搭载, 结合前期设定的飞行路线来 确定飞行的方向, 这样才可以对航拍的效果加以保证。将 POS 系统加以实践运用可以更加高效的对方位元素加以确定, 并且将摄影中心空间坐标进行记录, 确定飞行的姿态。将倾斜相机加以合理的运用, 能够确保拍摄的信息具有良好的 准确性, 这样才可以为不动产测绘工作的实施给予技术辅助 ${ }^{[1]}$ 。

(2) 在倾斜摄影测量技术中涉及到多个关键技术, 纵向摄影数据与倾斜摄影数据在多视影像中的作用是非常重要 的, 倾斜摄影数据要想借助传统空中三角测量系统来加以处理是非常困难的, 在实践操作中应当由专业人员对影像的 几何变形与遮挡关系加以切实的分析研究, 这样才可以保证影像联合评差能够达到良好的合理性。以 POS 系统为辅助, 以金字塔匹配策略为基础, 可以在所有的影像上自动搭配同名点, 自由网光束法平差就可以实现既定的效果目标, 同 名点匹配结果具有良好的效果。其次, 需要对控制点坐标, GPU/IMU 辅助数据的多视影像自检校区域网平差误差方程进 行建立, 利用专业的计算方法来对平差的结果的准确性加以保证。其次, 是多视影像密集匹配, 实际涉及到的范围较 
大, 并且分辨率相对较高, 应当保证在匹配的过程中对冗余信息进行综合分析, 确保多视影像同名点坐标信息的准确 性。在科学技术快速发展的过程中, 多基元、多视影像匹配要想保证良好的发展, 还需要进行不断的研究 ${ }^{[2]}$ 。结合多视 影像的搜索特征来说, 主要涉及到建筑结构边缘、墙体结构纹理等等, 确定二维矢量数据集, 促使影像的基本二维特 征朝着三为特征转变, 结合实际情况来对影像因子加以设置, 对于墙面加以确定, 并落实合理的分类, 这样就可以以 建筑墙体结构为对象来实施平面的扫描或者是分割, 这样就可以实现对建筑工程的侧面结构的重建。

\section{2 倾斜摄影测量技术主要特点}

\section{1 真实反应物体情况}

运用倾斜摄影测量技术所获得的信息数据更加的准确, 并且也能够对客观事物的实际情况加以反应, 因为这项技 术与以往老旧的人工建模技术更加的优秀, 所以受到了人们的广泛关注。在倾斜摄影测量技术准确性不断提升的形势 下，这项技术的适用范围也在逐渐的扩展。

\section{2 输出数据具有多样性}

倾斜摄影测量技术自身涉及到空间位置数据相对较多, 能够切实的缓解以往传统测量方式中所存在的各种问题, 借助这项测量技术进行建模, 可以有效的提高对建筑物纹理的掌握效率, 这样才可以带动数据处理的整体效果的不断 提高 ${ }^{[3]}$ 。

\section{3 倾斜摄影测量技术在不动产测绘中的应用}

\section{1 应用实例}

这篇文章主要围绕羊口镇三维大数据平台建设项目为基础, 针对倾斜摄影测量技术在不动产测绘中的实践运用进 行深入分析研究。

\section{1.1 倾斜摄影测量技术参数}

在倾斜摄影测量技术中最为关键的一个参数就是地面分辨率, 一般来说, 不同的比例尺的测绘图所需要的平均分 辨率都是不同的, 并且倾斜的角度的要求也是不一样的, 有效的航线要保证超出成图范围的六条基础线路, 旁向的重 叠度需要保证达到规定的标准, 摄像色彩需要确保良好的均衡、清晰, 颜色饱满, 不能存在云影或者是划痕的情况, 反差应当加以良好的保证。在针对照片数据进行存储和包装的时候, 照片的数据在飞行的过程中进行导出, 结合镜头 来自行进行导出, 还应当针对架次进行编号, 在针对相片颜色、质量以及编号进行检查之后, 结合项目和日期来完成 保存 ${ }^{[4]}$ 。

\section{1 .2 倾斜摄影测量准备工作}

在倾斜摄影工作实施之前, 为了确保工作的效率和效果应当结合实际需要来做好充分的准备工作。在飞行之前还 需要到相关部门进行申报, 并且在地方派出所进行备案, 尽可能的规避发生黑飞而出现被扣押的情况。飞机和机组人 员、摄影工作人员应当结合各方面实际情况来制定工作计划, 从而为后续各项工作的实施给予规范性的指导, 保证工 作的效率和安全。

\section{1 .3 倾斜摄影测量的实施}

技术人员应当结合环境情况以及其后情况来对各种恶劣天气进行综合分析, 并且做好切实的准备工作, 尽可能的 保证飞行的稳定性。在正式开始飞行之前, 还需要对摄影硬件进行检查, 保证设备始终维持在良好的状态, 这样才可 以切实的保证摄影的效果和质量。

\section{2 倾斜摄影测量技术在不动产测绘中的应用策略}

\subsection{1 飞行质量控制}

借助高性能的飞行控制设备来获得精准的飞行数据, 并且将 GPS 导航系统进行切实的运用, 尽可能的规避因为卫 星失锁而发生 GPS 导航失效的情况。

\subsection{2 摄影质量控制}

要想从根本上不断的提升摄影的效率和效果, 最为重要的就是需要技术人员不断的提升摄影质量控制力度, 并且 需要对下列几个方面加以侧重关注: 对于摄影的天气需要进行前期的了解, 并且尽可能的选择在晴天能见度较高的天 气进行拍摄, 保证地面不会受到云朵对太阳光的遮挡, 并且还要保证良好的光照度。结合飞行的情况以及光照等多方 面情况来对曝光系数进行合理的设计, 尽可能的提升摄影的效果和质量。确保提交的成果影像中单张彩色像片的清晰 
度能够达到规定的标准要求, 这样带可以通过照片更加准确的对地表中的所有事物进行判断, 从而对地物的进行绘制, 确保两两邻近的地物的色调保持一致性。在航拍工作结束之后, 技术工作人员应当运用飞行管理软件对于摄影站点坐 标数据进行专业的处理, 如果发现任何的差异, 应当进行补拍 ${ }^{[5]}$ 。

3.2 .3 做好像控点布设工作

首先, 像控点需要布设成平高点, 像控点需要根据区域的实际情况, 先布设目标范围周边, 再布设测区中间位置, 像控点布设距离需要控制为约 $300 \mathrm{~m}$ 。其次, 任意一个地块至少测量 5 个像控点, 并且包裹住测量区域, 如地形特殊, 确 保最大范围的包裹目标。

\section{4 结语}

总的来说，倾斜摄影测量技术是当前最为先进的一种测量技术，这项技术的出现有效的解决了以往传统摄影测量 技术中的问题。当下, 在实施不动产测绘工作的时候, 将这项技术加以实践运用, 对于提升工作的效率和效果起到了 重要的影像作用。但是在将这项技术加以使用的时候, 往往会受到外界多方面因素的影像, 所以在数据的计算上也会 呈现误差, 因此需要加强研究, 早日突破难题。

\section{[参考文献]}

[1]任国庆.倾斜摄影测量技术在不动产测绘中的应用策略 [J].住宅与房地产, 2020(21): 235,

[2] 游芳.倾斜摄影测量技术在不动产测绘中的应用策略 [J]. 工程建设与设计, 2020 (6) : 271-272.

[3]喻智华.分析倾斜摄影测量技术在不动产测绘中的应用 [J].价值工程, 2019, 38 (31) : 252-253.

[4]高勇良.倾斜摄影测量技术在不动产测绘中的应用 [J].地产,2019(9) : 47-48.

[5]喜文飞, 李国柱, 赵子龙, 白世睈, 张东升。倾斜摄影测量技术在不动产测绘中的应用研究 [J]。城市勘 测, $2019(1): 70-74$.

作者简介: 张萍 (1989.7-), 毕业院校: 安徽建筑工业学院土木工程学院, 所学专业: 土木工程专业, 当前就职单位: 山东金桥建设项目管理有限公司。 